\title{
La cumulativité en sciences sociales : distances entre les théories
}

Pierre Livet

\section{OpenEdition}

\section{Journals}

Édition électronique

URL : http://journals.openedition.org/ress/472

DOI : $10.4000 /$ ress.472

ISSN : 1663-4446

Éditeur

Librairie Droz

\section{Édition imprimée}

Date de publication : 1 janvier 2006

Pagination : 131-148

ISBN : 2-600-00959-0

ISSN : 0048-8046

\section{Référence électronique}

Pierre Livet, "La cumulativité en sciences sociales : distances entre les théories », Revue européenne des sciences sociales [En ligne], XLIV-133 | 2006, mis en ligne le 12 novembre 2009, consulté le 21 avril 2019. URL : http://journals.openedition.org/ress/472 ; DOI : 10.4000/ress.472 


\section{LA CUMULATIVITÉ EN SCIENCES SOCIALES : DISTANCES ENTRE LES THÉORIES}

Le processus qui est décrit ici concerne une dynamique qui permet à la fois l'épuration (exclure des classes d'explications), l'intégration (inclure de nouvelles explications), et la restructuration (changer l'ordre). Les deux premières opérations peuvent se ramener à l'idée de révision (contraction = épuration, extension après contraction = intégration) et la troisième exige de réviser l'ordre qui guide une révision. Il semble donc aisé d'appliquer la méthode d'analyse ici proposée aux analyses des rapports entre différents sociologues (Giddens est peut-être un cas délicat, car il ne définit pas clairement ses positions, mais pour Boudon, c'est plus facile, encore qu'il faille ajouter la prise en compte du «contexte» aux postulats posés par Berthelot). La méthode développée ici permet éventuellement de montrer que le degré de cumulativité peut varier entre le recueil des données, l'utilisation de différents modèles, les explications et interprétations, et les programmes proposés.

En outre, ce qui est proposé ici, c'est de profiter d'une correspondance entre syntaxique et sémantique, correspondance assurée au niveau des conditions de cohérence, pour nous réduire à la notion de classes d'explications (qui sont des classes de formules inférentielles). Cette notion permet d'intégrer les «données» (aucune donnée pure ne peut faire évoluer une théorie, il faut qu'elle apparaisse au moins comme contredisant une inférence explicative), les éléments «programmatiques » (c'est une sélection ordonnée d'un ensemble restreint de classes très générales d'inférences explicatives) et la résolution de contradictions syntaxiques et d'ambiguïtés sémantiques (qui ne donnent lieu à résolution que quand elles entraînent des contradictions). Les «modèles » sémantiques sont ici aussi ordonnés (on parle de «modèles préférentiels »).

Si je préfère partir d'une approche syntaxique, c'est qu'elle permet de s'appuyer sur des énoncés existants. Pour proposer une approche sémantique, je devrais, en vertu du holisme sémantique, proposer une structure globale de la sémantique d'une théorie sociologique, et je doute que l'on puisse y parvenir. Je préfère une méthode qui est opératoire et qui peut du coup donner lieu à discussions sur des textes, plutôt qu'une théorie générale qui n'est pas applicable telle quelle à une étude d'un corpus précis.

Walliser envisage à la fois la cumulativité additive (qui ajoute de nouveaux domaines, de nouvelles conclusions, de nouveaux outils théoriques, etc.) et la cumulativité évolutive par révision. C'est évidemment seulement la seconde qui m'intéresse, et il semble bien qu'en sociologie, ce soit la plus saillante. La première risque de se réduire à ce que Berthelot appelle une cumulativité par adjonction, sans que les sociologues se soucient trop de savoir si les nouvelles 
données sont cohérentes avec les explications dans des champs précédents. Je suggère qu'il n'est pas souhaitable d'accentuer les différences entre théories sociologiques, et donc qu'il faudrait réduire au maximum le type de distance (de degré de violation d'une stricte cumulativité) que je propose et pour lequel je donne des procédures d'évaluation. Mais inversement, il me semble qu'il serait souhaitable d'accentuer les différences en économie, où l'on a tendance à les supposer réduites un peu vite, au détriment d'une prise en compte des révisions que pourraient introduire la prise en compte de nouveaux domaines. Il faudrait donc utiliser la même méthode pour éviter une réduction des distances hâtive, ou au contraire pour vérifier qu'elle a bien été opérée avec efficacité.

\section{CUMUlativité ET CONDITIONS DE COHÉRENCE}

La cumulativité ne consiste pas forcément à toujours ajouter et ne jamais rien retirer. Ce serait absurde dans le domaine des sciences historiques. Cela n'est même pas réalisé pour les sciences physiques; le domaine de validité de la théorie de Newton est restreint par la théorie d'Einstein. Nous devons donc admettre des révisions de théories. Mais nous devons alors concevoir une notion de cumulativité qui soit compatible avec ces révisions.

Un premier essai nous est donné par les conditions dites de «cohérence » entre deux théories dont la seconde est une révision de la première. Une première situation possible est celle d'une première théorie incohérente tandis que la seconde restaure la cohérence en éliminant quelques propositions. Mais le cas qui nous intéresse le plus est celui où la seconde théorie tient compte d'une nouvelle donnée expérimentale dont la première ne disposait pas, et qui est en contradiction avec les conclusions des hypothèses de la première théorie.

Ces conditions ont été formulées d'abord par les théoriciens du choix social (pour définir le propriétés souhaitées pour une fonction de choix collective), sans rapport avec la notion de révision. Elles ont été retrouvées indépendamment par les théoriciens des opérations de révision. Cela donne à penser que ce sont des conditions importantes pour penser une notion de cohérence à travers des modifications, intégrations, transformations et révisions. Elles doivent donc pouvoir nous donner des indications sur les exigences associées au concept de cumulativité que nous souhaitons identifier, qui est celui d'une cumulativité à travers des changements de théorie. Notre but final n'est pas de disposer d'un seul concept de cumulativité, mais au contraire de plusieurs concepts, gradués entre eux selon que leurs exigences sont plus fortes ou plus faibles. Nous pourrions ainsi proposer une forme de quasi-distance entre des théories.

\section{2. Problèmes de méthode}

Mais avant d'aller plus loin, il nous faut poser et tenter de résoudre des problèmes de méthode Une objection à toute référence à ces conditions de « cohérence» est que leur formulation implique des fonctions qui vont d'un ensemble dans l'ensemble de ses parties ${ }^{1}$. En effet, une fonction de choix social doit choisir

\footnotetext{
Cette objection vient de Walliser.
} 
entre des choix individuels ou bien faire une sorte de synthèse des choix individuels, et ces choix sont déjà une manière de ranger ensemble certains des éléments d'un ensemble d'options. Les fonctions de choix ou de sélection sont donc des fonctions de l'ensemble E dans l'ensemble des parties de E. La sémantique des mondes possibles utilise de même la grande richesse de cet ensemble des parties de E. Mais la condition pour que ces fonctions de choix aient un sens est alors que les éléments ainsi rassemblés soient mutuellement exclusifs et qu'ils épuisent, pris collectivement, la totalité de l'ensemble. Cette condition semble difficile à assurer dans le cas des propositions des théories sociologiques.

Nous pouvons alors prendre un détour. Ces conditions de cohérence correspondent aussi à des façons d'ordonner les choix (quand on s'intéresse à un choix collectif) ou, dans le domaine de la révision, d'ordonner les modèles qui donnent la sémantique d'un énoncé. Elles impliquent chacune des structures d'ordre. Une fonction de choix ou de sélection consiste à retenir les modèles «minimaux », ce qui veut dire, les modèles prioritaires, donc à introduire un certain ordre.

Par ailleurs, quand nous considérons une formule qui comporte une inférence, cette inférence peut être valide logiquement, ou bien n'être valide que moyennant des connaissances spécifiques, qui sont liées à nos estimations de ce qui est plus ou moins normal ou plus ou moins fréquent dans le domaine. Quand une inférence n'est pas valide logiquement mais nous paraît normale, cela révèle donc quelque chose sur l'ordre implicite de nos croyances. Soit l'énoncé: «si nous avons comme données: tel individu est catholique et/ou il a de fortes chances de se suicider, alors nous considérons comme normal qu'il soit catholique, mais pas qu'il ait de fortes chances de se suicider». C'est un énoncé qui a la forme logique «de (A ou B) inférer normalement $(\mathrm{A}$ et non $\mathrm{B}) »$. Or « $\mathrm{A}$ et non $\mathrm{B}$ » est plus fort logiquement que « $\mathrm{A}$ ou $\mathrm{B}$ ». Mais en logique, la conclusion ne doit pas avoir une force logique plus grande que la moins forte des prémisses. Quand donc nous acceptons une inférence dont la conclusion est plus forte logiquement que les prémisses, soit nous faisons une erreur de raisonnement, soit, si nous avons de bonnes raisons de persister à trouver notre inférence plausible, nous révélons que A a pour nous une priorité sur $\mathrm{B}$ (c'est là un résumé de nos bonnes raisons). Il peut s'agir d'une préférence épistémique portant sur l'inférence, ou du fait que nous tenons la conclusion pour plus normale et plus plausible. Cet ordre révélé reste local: il ne porte que sur A et $\mathrm{B}$, et dans un domaine D. Mais c'est une donnée dont devrait tenir compte une fonction de choix ou de sélection qui retient seulement certaines explications comme admissibles. Or cet ordre nous est fourni en restant au niveau des formules, ou plus exactement des relations locales d'inférence entre les formules, sans avoir pour l'instant à rentrer dans une sémantique des mondes possibles.

Mais si chaque inférence qui manifeste une force de la conclusion supérieure à celle des prémisses nous révèle un ordre particulier, les ordres locaux révélés par diverses inférences n'ont aucune raison de former entre eux un ordre global cohérent. Or les conditions de cohérence dues aux économistes et que nous retrouvons dans les théories de la révision sont des conditions qui assurent le maintien de certaines relations entre ces ordres locaux, voire régionaux, par exemple entre l'ordre qui résulte d'un ensemble de propositions limité et celui qui résulte d'un ensemble de propositions plus large et qui inclut le précédent. Ce sont des conditions sur le passage d'un ordre régional à un ordre global, ou d'un ordre régional à celui d'une autre région. 
Dès lors, considérons des inférences tirées d'une théorie sociologique. Nous pourrons sans doute montrer que certaines de ces inférences révèlent un ordre autre que logique, un ordre qui reste local ou régional, limité à ces inférences. Il est possible que certaines inférences que nous rencontrons dans la théorie $\mathrm{T}$, et qui révèlent un certain ordre, se retrouvent dans la théorie T'. Imaginons alors que la théorie T' nous propose aussi au moins une inférence qui n'est pas validée en T. Supposons que chacune de ces inférences révèle un ordre de priorités, et que la nouvelle inférence nous contraigne à intervertir au moins une des relations d'ordre révélées par les inférences que nous retenons de la théorie $\mathrm{T}$, voire à exclure une classe d'inférences dont le rang était élevé dans la théorie T. Ce changement est incompatible avec une cumulativité stricte, puisqu'une révision importante a eu lieu. Nous nous tournerons alors vers les conditions de cohérence, et nous pourrons nous demander, en nous réduisant à ces ensembles d'inférences et aux ordres révélés, si elles sont malgré tout satisfaites.

Notre analyse sera évidemment limitée à ces inférences communes entre les deux théories et à ces inférences qui intervertissent l'ordre propre à une première théorie. Elle ne peut pas prétendre nous donner une comparaison entre les deux théories dans leur ensemble, ni entre l'ensemble des modèles de ces deux théories, ni entre les ensembles de mondes possibles qui constitueraient la sémantique de ces théories. Mais elle nous permet de saisir les points sensibles des relations entre les deux théories, ceux pour lesquels les problèmes de cumulativité se posent de manière significative. Et pour ce faire elle n'a besoin que d'un ensemble limité de formulations d'inférences.

Heureusement, car si nous devions, pour définir des relations entre les théories, les considérer dans la totalité de ce qu'elles affirment, nous devrions disposer d'une sémantique complète pour chaque théorie, et donc savoir l'ensemble des modèles qu'elles falsifient et qu'elles valident, ce qui est définitivement hors de notre portée.

Mais par chance il n'est pas nécessaire de construire une sémantique pour chaque théorie, de savoir sur quels ensembles de mondes possibles nous ouvrent nos inférences (ce dont nous serions bien incapables). En effet, nous savons (Rott 2001, Schlechta 2004) qu'il y a une correspondance simple entre 1) les conditions de cohérence formulées dans le domaine du choix social, 2) leur formulation dans une théorie de la révision qui implique une sémantique des mondes possibles, 3 ) leur formulation dans une sémantique algébrique qui repose essentiellement sur une notion de l'importance d'un sous-ensemble par rapport à un ensemble, 4) leur formulation dans les termes d'une comparaison entre des «théories» au sens logique (à savoir l'ensemble de toutes les conséquences d'un énoncé selon les axiomes et les règles de la théorie), et finalement 5) leur définition portant simplement sur des relations d'inférence plausible entre des formules, donc des énoncés.

Mais comme on va le voir, ce qui nous intéresse, c'est de considérer les cas où ces conditions de cohérence ne sont pas satisfaites, et où cependant la transition d'un ensemble d'inférences à un autre ensemble nous paraît avoir de solides justifications. On devine à quelle stratégie nous allons recourir: de même que, lorsque les relations de force logique entre prémisses et conclusion ne sont pas respectées et que l'inférence nous paraît pourtant plausible, cette distorsion révèle un ordre de priorité, de même une violation des conditions de cohérence qui reste plausible nous révèle un ordre de priorité, mais cette fois non pas entre les formules ou les énoncés, mais entre les théories. 
Or les ordres de priorité que nous révélaient nos inférences logiquement audacieuses avaient entre autre pour fonction de guider nos révisions (quand il fallait réviser, nous abandonnions les propositions les moins prioritaires et conservions les autres). Mais ces révisions supposaient alors que soit donné et fixe l'ordre qui les guidait. A présent, nous sommes montés un niveau plus haut. Nous avons maintenant affaire à une révision de l'ordre de priorité propre à une théorie T, révision qui nous donne l'ordre de priorité propre à la théorie suivante T'. Les violations des conditions de cohérence que nous considérons cependant comme plausibles nous révèlent donc les changements de priorités que nous acceptons.

Mais à quelle condition est il rationnel d'accepter de tels changements? Quand il s'agit d'une révision de premier ordre, il est rationnel de réviser quand nous avons une nouvelle information fiable qui contredit nos conclusions. Maintenant qu'il s'agit d'une révision de l'ordre qui guide les révisions de premier ordre, il sera rationnel de réviser quand nous détecterons une incohérence entre l'ordre révélé par les inférences de la théorie T, et les inférences révélées par la théorie T', mais à condition que cette incohérence soit liée à des éléments nouveaux: informations nouvelles fiables, hypothèses nouvelles, cohérentes avec ces nouvelles informations, et qui nous permettent de nouvelles inférences et nous donnent de nouvelles conclusions qui nous font encore découvrir d'autres informations supplémentaires.

Plus précisément, il semble raisonnable de n'accepter comme justifié un changement de priorités qu'aux conditions suivantes:

1) Nous devons pouvoir trouver dans la théorie T' une information nouvelle I*, fiable mais incohérente avec une des conclusions de la théorie $\mathrm{T}$ (trouver une incohérence dans la théorie $\mathrm{T}$, sans information nouvelle, aurait amené une révision, mais qui aurait simplement supprimé des inférences et des conclusions sans changer l'ordre).

2) Cette information nouvelle $I^{*}$ donne lieu à révision, et la première étape de cette révision est forcément traitée selon l'ordre de priorité de la théorie initiale T. Elle aboutit donc à supprimer un ou des éléments de T qui ont le rang de priorité le plus bas.

3) Pour que l'ordre de priorité de T soit changé, il faut donc qu'à la suppression de ces éléments, nous joignons les nouvelles inférences que permet la combinaison de $I^{*}$ avec des propositions de rang supérieur, et leurs conclusions. Dans l'ensemble d'inférences et de conclusion ainsi modifié, il se peut que des inférences qui avaient auparavant une priorité faible (elles n'étaient qu'exceptionnellement valides) deviennent normales, et inversement, et cela parce que nous avons enlevé des conclusions qui bloquaient certaines inférences, et que nous en avons ajouté qui en permettent d'autres. Et pour pouvoir exprimer ces changements de priorité, et les justifier, il nous faut disposer de ce que nous pouvons appeler des méta-inférences, du genre: «si les inférences de la classe C qui était prioritaires sur celles de la classe C' n'ont de conclusions valides que dans des domaines exceptionnels, donc non prioritaires, et que les inférences de C' sont toujours valides de manière non exceptionnelle, alors normalement les inférences de $\mathrm{C}$ ne sont plus prioritaires par rapport à celles de C'». 
4) Il faut que nous puissions non seulement dégrader le rang de priorité des inférences en conflit avec la nouvelle information, mais que nous puissions exclure certaines de ces inférences. C'est nécessaire si nous voulons dans la nouvelle théorie $\mathrm{T}$ ' éliminer les incohérences avec certains éléments de la théorie T. Il doit bien y avoir des propositions qui ne seront pas simplement considérées comme valides seulement par exception, mais comme non valides. Il se peut que cette exclusion ne soit pas possible pour des inférences normales de base, puisque même si nous leur donnons un rang de priorité inférieur, elles pourront toujours être valides par exception. En revanche nous pouvons procéder à une telle exclusion pour les méta-inférences, qui soutiennent que telle inférence est toujours valide, ou normale, ou exceptionnelle, bref qui portent sur l'ordre de priorité lui-même. Nous allons donc pouvoir éliminer certaines des méta-inférences de la théorie T.

5) Le processus de transition doit être graduel. A chaque étape, nous justifions au maximum un seul changement d'ordre.

Si nous arrivons à respecter ces conditions pour justifier une transition entre deux théories, étapes par étapes, il nous restera à voir combien d'étapes sont nécessaires pour justifier une transition qui constitue la violation d'une condition de cohérence, puis d'une condition de cohérence plus exigeante, et ainsi de suite. Si nous parvenons à montrer que plus il y a d'étapes dans notre chemin de transition justifiée, plus sont fortes les conditions de cohérence dont nous pouvons justifier la violation, nous pourrons penser disposer d'un processus qui nous permettrait d'extraire de l'examen des deux théories une notion de quasi-distance entre les théories (évidemment pas une mesure de cette distance). Nous pourrions alors remplacer le concept de cumulativité, qui est un concept par tout ou rien, par un concept de distance, qui est un concept suceptible du plus et du moins, et qui permet d'ordonner deux distances entre elles. Selon que la distance entre les deux théories serait supérieure ou inférieure, le rapport entre deux théories serait donc plus ou moins cumulatif. Notre notion de distance correspondrait donc à une sorte de degré de violation des conditions de cohérence, quand cette violation est justifiée, étape par étape. Si nous devons accomplir plus de violations justifiées pour passer d'une théorie à une autre, que pour passer à une troisième, la distance sera supérieure. En résumé, une distance supérieure entre théories correspond à une violation plus importante de la cumulativité au sens restreint et des conditions de cohérence qui définissent des formes de cumulativité plus laxistes. Mais tant que nous pouvons définir cette distance, nous pouvons tout de même parler de cumulativité, même si cette cumulativité est de plus en plus faible.

\section{DURKHEIM ET DOUGLAS}

\subsection{Leurs propositions}

Prenons donc un exemple en sociologie. Comparons la théorie du suicide de Durkheim et celle de Douglas. Selon Durkheim, il ne faut pas tenir compte dans l'explication sociologique des suicides des raisons avancées par les individus, ni même d'éventuelles causes pathologiques (d'un tempérament individuel maladif, 
ce que nous désignerons par TM) mais des faits collectifs donnés par les statistiques. On peut alors expliquer les variations des statistiques, et ce d'abord en fonction du degré d'intégration sociale de l'individu. Par intégration, il faut entendre une forme d'attraction de l'individu par la société, qui fournit à l'individu les représentations collectives et les raisons de ses interactions adossées à un dense réseau de relations sociales. L'influence de l'intégration sur le suicide prend la forme d'une courbe en U. Quand l'intégration est très forte, on observe le suicide «altruiste», l'individu estimant sa vie de peu d'importance face aux besoins sociaux. Quand elle est très faible, que l'individu se sent individuellement et isolément en charge de ses propres significations, l'appui de l'intégration sociale lui manque, et on observe le suicide «égoïste». Durkheim ajoute un second facteur, la régulation ou pression normative sociale. C'est forcément dans le cas du suicide un facteur de second rang, puisque si l'individu n'est pas intégré, la pression ne peut pas être très efficace. En revanche, la pression régulatrice peut être forte sans que l'intégration le soit, quand la société impose des sanctions mais ne fournit pas de significations positives aux projets individuels. Dès lors, si la régulation sociale n'oppose pas aux désirs et passions individuelles une pression suffisante, nous aurons le suicide par anomie. Si elle est trop forte, un suicide fataliste.

Cette théorie va donc énoncer des propositions du genre «si x est catholique, $\mathrm{x}$ a une probabilité statistique de se suicider (par suicide égoïste) inférieure à celle de y qui est protestant». Nous savons pouvoir ramener ce genre de proposition à une suite d'énoncés du genre «(x est catholique et il a une probabilité statistique $\mathrm{Pr}>\mathrm{pr}$ ) et/ou (x est catholique et il a une probabilité statistique $\mathrm{pr}<\mathrm{Pr}$ de se suicider) implique normalement (x est catholique et il a une probabilité statistique $\mathrm{pr}<\mathrm{Pr}$ de se suicider) et non (x est catholique et il a une probabilité statistique $\mathrm{Pr}>\mathrm{pr}) »^{2}$; inversement pour les protestants; «X est catholique implique normalement $\mathrm{x}$ a une intégration Int $>$ int», «x est protestant implique normalement $\mathrm{x}$ a une intégration int<Int», etc. Tout cela est fastidieux, et nous allons résumer toute cette classe d'inférences par le sigle IS, pour «intégration sociale».

De même, nous aurons une classe d'inférences RS (pour « régulation sociale») qui affirme entre autres que « $\mathrm{x}$ est catholique implique normalement $\mathrm{x}$ est soumis à une pression de régulation $\mathrm{Reg}>\mathrm{reg}$ », et inversement pour un protestant. Nous avons alors un problème, qui est que des inférences de la classe IS se retrouvent dans celles de la classe RS, puisque RS nous permet aussi d'affirmer que les probabilités de suicide sont plus élevées pour les protestants que pour les catholiques. Mais ce sont là des conclusions. Les prémisses ne portent pas sur l'intégration, mais sur la pression régulatrice, du genre «si (x est catholique) et/ou (la pression régulatrice de son milieu est faible), alors normalement, (x est catholique) et (la pression régulatrice de son milieu est forte)». Cependant, si l'individu n'est pas intégré, la pression régulatrice semble devoir être moins efficace. Si nous partons d'un individu mal intégré car protestant, qui se trouve par ailleurs dans une société de plus forte pression (mettons, sans possibilité de changer faci-

2 La formulation que nous donnons revient à présenter les prémisses des inférences sous une forme normale disjonctive (une disjonction de conjonctions) et à écrire les conclusions sous une forme qui mette en évidence une conjonction de la négation d'une de ces prémisses disjointes, et des autres prémisses. 
lement de pays), ses probabilités de suicide seront encore élevées. Ce que nous pouvons résumer en disant que «si (IS faible et RS fort) et/ou (les chances de suicide sont faibles), alors normalement (les chances de suicides ne sont pas faibles)». Cela veut dire qu'entre les conclusions que nous tirons par une inférence normale de IS (chances de suicide faible) et celles que nous tirons par une inférence normale de RS (chances fortes) nous allons rejeter les secondes et choisir les premières. Et nous révélons ainsi que nous assignons aux prémisses IS une force inférentielle supérieure à celle des prémisses RS, donc que si une révision était nécessaire, nous sacrifierions les inférences en RS et pas celles en IS, ou encore que IS est prioritaire par rapport à RS.

Le fait que les conclusions des inférences en IS et les conclusions des inférences en RS soient mêlées n'est donc pas un handicap pour notre approche. C'est tout le contraire. C'est indispensable pour nous permettre d'ordonner entre elles ces classes d'inférence.

Est-ce que ces formulations expriment bien la théorie de Durkheim? Nous avons déjà dit qu'il nous est impossible, comme à quiconque, de vérifier qu'elles expriment toute la théorie de Durkheim. Et d'ailleurs ce n'est pas du tout notre but, puisque pour notre propos, il nous suffira de trouver quelques inférences communes entre Durkheim et Douglas et au moins une inférence de Douglas incohérente avec celles de Durkheim. Par ailleurs, celui qui prétendrait que même une fois cette limitation reconnue nos formulations sont infidèles à cette théorie devrait alors proposer d'autres formulations, en les appuyant sur les textes de Durkheim. C'est très probablement possible. Mais tant que cette révision n'est pas proposée, nous supposons par défaut que nos formulations sont cohérentes avec la théorie de Durkheim. De toute manière, aucune formulation ne pourra avoir de prétention plus élevée que cette cohérence par défaut. Nos analyses actuelles restent d'ailleurs approximatives, et notre but est seulement de voir comment mettre au point une méthode à partir d'un exemple.

Venons-en à Douglas. Il introduit une nouvelle donnée: les statistiques dépendent des choix des officiels qui décident de déclarer comme suicide telle ou telle mort (appelons ces déclarations sociales DS). Cela, dit Douglas, jette un doute sur les explications sociologiques trop générales (ces explications, nous les désignerons par EGA: explications générales abstraites de tout contexte particulier). Il vaut donc mieux faire une analyse plus fine des raisons pour lesquelles les gens se donnent la mort. Mais ce sont là des significations socialement construites (des patterns socialement significatifs: PS). De plus, la mobilisation de ces significations dépend du contexte particulier de faits, d'histoire passée, et d'autres significations sociales mobilisées dans lequel celui qui communique - que ce soit celui qui veut se suicider, son entourage ou les officiels- se trouve plongé.

\subsection{Comparer les théories}

Quels rapports pouvons nous faire entre les deux théories? Douglas range évidemment les explications de Durkheim dans la classe des EGA. Les explications qui proposent des facteurs définis seulement au niveau statistique sont générales et abstraites. Or la classe des inférences DS nous dit que les statistiques dépendent de ceux qui déclarent les morts dans la rubrique des suicides ou sous d'autres rubriques. Et les raisons de ces officiels sont individuelles, ce ne sont pas 
des faits statistiques. Donc dans la mesure où les explications de Durkheim sont des EGA, Douglas soutient que «DS ou EGA» implique normalement «DS et non EGA». Si les explications de Durkheim sont effectivement des EGA, alors voilà une inférence ou une classe d'inférence avancée par Douglas qui donne des conclusions incohérentes avec les explications de Durkheim ${ }^{3}$.

Mais, ajouterons-nous, Douglas ne peut pas éliminer le facteur d'intégration sociale, même s'il le trouve trop vaguement défini. En effet, il reconnaît lui-même que les officiels chargés de déclarer le suicide peuvent être influencés par le fait que le mort était soit un membre influent du groupe social dont ils font partie, soit un parent d'un membre influent, et dès lors ces officiels vont tenter de ranger la mort dans la catégorie qui leur semble convenir à la catégorisation souhaitée dans ce groupe. Ainsi les modes d'intégration sociaux ont bien toujours une influence sur les déclarations de suicides. Ce qu'exclut effectivement Douglas, c'est que cette explication par l'intégration puisse se fonder sur les statistiques. Et si les statistiques sont utilisées comme un filtre permettant de ne retenir que des explications générales non contextuelles et pas des explications qui s'appuient sur les motivations contextuelles des individus, guidés par les patterns socialement significatifs qu'active pour eux tel contexte, alors les corrélations que permettent de dégager leurs données sont prises pour des EGA. Mais l'intégration sociale peut tout aussi bien fonctionner au niveau de l'individu en contexte. Douglas pense donc qu'une explication par l'intégration sociale est valide. Ce qu'il critique, c'est l'idée d'une forme homogène et générale d'intégration, valide pour tout un groupe. L'intégration effective est seulement une modalité particulière de l'efficace des patterns socialement significatifs, qui varient à l'intérieur d'un même groupe. Donc si, sans avoir d'informations plus précises, nous avons «PS ou IS », Douglas retiendra normalement PS et rejettera IS, parce que les patterns sociaux tiennent compte de ces variations, alors que l'intégration sociale ne propose pas explicitement d'en tenir compte. Mais il ne l'exclura pas des modes explicatifs qu'il retient, alors qu'il exclura les $\mathrm{EGA}^{4}$.

De même, les régulations ou pressions sociales RS ne constituent qu'un élément minoritaire des PSI. En effet, ces patterns reconnaissent que celui qui se sent désespéré et qui veut lancer un appel au secours par sa tentative de suicide, ou se venger du sort indigne qu'il a subi, a des raisons socialement significatives de se suicider, alors même que les normes sociales considèrent cette forme d'appel au secours comme absurde, et cette recherche de vengeance comme déraisonnable, voire les deux comme religieusement condamnables. Ainsi les RS ne sont pas prioritaires pour Douglas. Mais rien ne nous permet de supposer que Douglas

Douglas émet un autre reproche, qu'il pense fondamental: Durkheim et ses successeurs présupposeraient l'homogénéité des formes aussi bien normatives qu'intégratives propres à la culture d'un groupe (The social meanings of suicide, p. 155). Si cette présupposition était une forme de dogme irrévisable, elle serait critiquable. Mais si c'est simplement une hypothèse par défaut, nécessaire pour commencer la recherche, et que l'on révisera seulement quand on aura constaté des différences, elle semble inévitable.

${ }_{4}$ Des études statistiques plus fines pourraient mettre en évidence non pas les contextes, mais les différences entre les contextes dans lesquels se trouvent les officiels qui déclarent ces statistiques. On retrouverait alors des EGA. Le problème reste cependant que recourir à d'autres éléments statistiques dépend toujours de ceux qui déclarent les données dont l'agrégation constitue ces éléments. 
récuse l'existence de normes sociales et de leur force régulatrice, via les patterns socialement significatifs. Nous devrons aussi les admettre dans sa théorie, mais à un rang inférieur.

Puisque nous nous demandons quelles révisions sont justifiées quand nous passons de Durkheim à Douglas, nous devons non seulement considérer ce que Douglas retient de Durkheim, mais aussi ce que Durkheim pourra accepter de Douglas. En effet, la relation de révision doit se penser dans les deux sens. D'une part, en partant de la première théorie, celle de Durkheim, nous devons nous demander quelles nouvelles données, ou quelles incohérences détectées et corrigées, nous donnent la seconde théorie, celle de Douglas, comme une révision de la première. Mais nous devons aussi nous demander dans quelle mesure la première théorie présentait des dispositions aux révisions faites par la seconde. Il se peut en effet que les nouvelles données ou les corrections d'incohérence amènent à distinguer des catégories chez Douglas là où Durkheim ne le faisait pas, et à ajouter des nouveaux domaines comme extensions des catégories ainsi distinguées. Mais nous devons alors nous demander si Durkheim n'aurait pas récusé l'introduction de ces distinctions et de ces nouveaux domaines. Ainsi, après avoir relu Douglas à partir de Durkheim, nous devons aussi relire Durkheim à partir des catégories de Douglas, pour voir si ces catégories pourraient se présenter comme des extensions ou bien des spécifications des catégories de Durkheim, ou non.

Nous devrons ainsi nous demander si Durkheim exclut les explications par l'intégration sociale ou la régulation sociale qui ne seraient pas filtrées par les statistiques et qui ne seraient pas des explications générales abstraites. Une telle interprétation semble exagérée, puisque Durkheim ne borne pas ses données aux statistiques. Ce qu'il exige, c'est que les explications recourent à des faits sociaux, et la marque du social, c'est le collectif. Mais par ailleurs Durkheim reconnaît que les faits sociaux impliquent des représentations, et que les individus partagent des représentations collectives. Comme les patterns socialement significatifs de Douglas sont sûrement des représentations qui sont partagées, et qui ne sont pas seulement des idiosyncrasies d'individus singuliers, nous pouvons les compter au nombre des représentations collectives. Mais comme Durkheim nous enjoint de ne pas tenir compte des représentations individuelles en tant que telles, mais seulement des représentations collectives des individus, nous devons donc faire une différence, parmi les PS, entre les patterns sociaux tels qu'évoqués par un individu, et les patterns sociaux proprement collectifs, entre les PSI et les PSC. Les PSC sont par exemple des coutumes collectives, des rites, etc.

Pour pouvoir comparer les classes d'inférences explicatives de Durkheim et Douglas, il nous faut donc procéder à un redécoupage des classes d'inférence de Durkheim en fonction des catégorisations de Douglas, et réciproquement, comme nous venons de le faire. Le principe pourrait en être le suivant: quand les classes d'inférence explicatives se chevauchent, nous prenons l'intersection du chevauchement comme une classe plus spécifique, et nous redéfinissons à partir de là les deux classes différentes qui incluent cette classe spécifique. Par exemple les PS se chevauchent avec les modes d'intégration en général, ceux qui pourraient aussi impliquer des EGA. Nous prenons donc les modes d'intégration qui sont aussi des PS (notre sigle IS vise en fait uniquement ces modes d'intégration, donc la restriction a déjà été faite), et nous redéfinissons la classe des modes d'intégration qui ne sont pas des PS comme rentrant dans la classe des EGA. Comme ce que nous 
redécoupons, ce sont des classes d'inférences explicatives, un tel redécoupage va permettre des méta-inférences (du genre, «si c'est une inférence explicative IS, normalement elle est non EGA», etc.).

Nos analyses nous permettent aussi de considérer qu'entre ces classes ainsi redécoupées, Durkheim propose un certain ordre, et Douglas un autre. Nos analyses requièrent pour Durkheim avec l'ordre 1) EGA, 2) IS, 3) RS. La théorie de Durkheim, par ailleurs, exclut les TM (tempéraments maladifs) et les PSI, et ne retient que les PS qui sont aussi des EGA, des IS ou des RS. L'ordre de Douglas serait le suivant: 1) PSI 2) IS 3) DS et RS. IS reste prioritaire par rapport à DS parce que dans les PSI, les motifs d'intégration vont rester importants, même s'ils sont plus variés que chez Durkheim, alors que les motifs purement idiosyncrasiques des officiels qui font les déclarations de suicides ne nous intéressent pas. Les RS, comme on l'a vu, peuvent être dominantes pour les officiels dans leurs déclarations, mais pas pour les suicidés. Elles sont donc d'un rang inférieur aux IS. Douglas exclut les EGA. Les PSC qui sont retenus sont soit des IS, soit des RS.

\subsection{Violation d'une condition de cohérence}

Cette analyse nous permet de nous demander si les conditions de cohérence que nous évoquions en commençant sont satisfaites ou pas.

La condition de cohérence la plus basique est la condition qu'en théorie du choix Sen a nommé condition a, (ou encore condition d'Hérédité, selon Aleskerov) et qu'on a nommée condition de préférentialité pour les logiques non monotones et les théories de la révision. En termes de modèles, si un modèle $m$ n'est pas minimal ou préféré dans l'ensemble $M$ des modèles d'une théorie $T$, alors, dans l'ensemble M' d'une théorie T' qui inclut $M$, il ne sera pas minimal non plus. Représentons nous la fonction de sélection $\mathrm{f}(\mathrm{S})$ qui choisit les modèles minimaux dans l'ensemble $S$, et sa consœur f(S'), qui les choisit dans l'ensemble $S^{\prime}$. Notre condition se formule alors : si $S \subseteq S$, alors $f\left(S^{\prime}\right) \cap S \subseteq f(S)$.

Si nous considérons des ensembles quelconques, $f\left(S^{\prime}\right) \cap S \subseteq f(S)$ nous donne la propriété dite de subexpansion. La condition est donc violée si la fonction de sélection sur S', quand nous l'appliquons sur S, y retient un élément que la fonction de sélection $\mathrm{f}(\mathrm{S})$ ne retenait pas (qui n'était pas minimal dans les modèles $\mathrm{M}$ liés à la théorie qui partait des données $\mathrm{S}$ ).

Dans notre analyse, ce qui correspond aux «modèles minimaux », ou ce que retient notre fonction de sélection, ce sont des classes d'inférences explicatives prioritaires $^{5}$. La théorie de Durkheim envisage différentes classes d'explications: PSI, IS, RS, EGA, TM. Elle retient \{EGA, IS, RS \}. Douglas envisage une classe de plus, DS. Nous pouvons donc prétendre que l'ensemble S' de Douglas inclut l'ensemble $S$ de Durkheim. Sur cet ensemble S, Douglas retient \{PSI, DS, IS, RS $\}$. Sa fonction de sélection ajoute donc PSI à ce que retenait $f(S)$. Nous avons donc là une violation de la condition a et de la condition de subexpansion.

Autrement dit, entre les théories de Durkheim et de Douglas sur le suicide, une condition de cohérence entre une théorie et sa révision n'est pas satisfaite. Il s'agit pourtant de la condition la plus faible.

\footnotetext{
5 Donc des classes de formules inférentielles.
} 
Cependant, la transition entre Durkheim et Douglas semble reposer sur des arguments assez solides proposés par Douglas. Durkheim n'a pas tenu compte des biais possibles dans les déclarations de morts comme suicides (DS). Cela diminue de beaucoup la pertinence de ses explications. Il faut donc recourir à d'autres données. Parmi elles, nous avons les patterns significatifs que les candidats au suicide empruntent pour rendre leur action socialement compréhensible (PSI). Mais les PSI ne sont vraiment explicatifs que dans le contexte particulier de chaque suicide, ou de chaque déclaration de suicide. Donc la sociologie ne peut pas prétendre à des explications générales abstraites, mais doit plutôt classer les significations socialement construites auxquelles les individus recourent dans des contextes d'usage particuliers (et exclure les EGA).

Pour rendre compte de l'aspect raisonnable de cette révision de Durkheim par Douglas, il nous faut donc nous demander s'il n'existerait pas des conditions de «trans-cohérence», c'est-à-dire des conditions qui permettent de justifier certaines violations des conditions de cohérence. Comme nous l'avons dit en introduction, notre hypothèse est que ces violations se justifient si nous avons des raisons de changer l'ordre des priorités quand nous passons de la théorie de Durkheim à la théorie de Douglas. Et notre seconde hypothèse est, si on la résume, que pour avoir le droit de changer d'ordre, il faut pouvoir le faire par des étapes qui doivent chacune être justifiées, 1) d'abord par une information nouvelle et fiable, mais en contradiction avec une des conclusions de la théorie précédente, 2) ensuite par les inférences que nous permet, en cascade, l'introduction de cette information en combinaison avec les inférences déjà existantes dans la théorie, et 3) finalement par les méta-inférences que nous pouvons faire sur la cascade de ces nouvelles inférences. Ces méta-inférences constatent que des conclusions jusque là normales sont devenues exceptionnelles, et nous permettent de juger que les inférences qui y conduisent ne sont plus normalement valides, mais seulement exceptionnellement valides, et qu'elles ne peuvent donc plus être prioritaires, si bien que l'ordre en est changé.

\section{LA TRANS-COHÉRENCE PAR ÉTAPES}

Par rapport aux conditions de cohérence classiques, nous introduisons une nouveauté: nous prenons en compte l'ordre des priorités entre les classes d'inférences explicatives dans une théorie, et c'est d'un changement de cet ordre que nous voulons rendre compte. Les conditions de cohérence, elles, tiennent compte simplement de la structure de l'ordre (est ce un pré-ordre, une structure par rangs, etc.).

Notre problème est maintenant, sur notre exemple, de savoir comment, en partant de la nouvelle donnée introduite par Douglas, DS, l'influence des motivations des officiels sur la déclaration des suicides, nous pouvons parvenir à changer l'ordre propre à la théorie de Durkheim. Comment passer de \{EGA, IS, RS $\}$ PSI, TM, à \{PSI, IS, DS et RS \} EGA, TM?

1) Nous introduisons la donnée conflictuelle DS. Nous avons donc \{EGA, IS, RS, DS \} PSI, TM. DS attaque directement la validité des inférences EGA. Mais puisque nous avons admis que nous procédions par étapes minimales, DS est 
pour l'instant au dernier rang. Les biais dans les déclarations peuvent donc pour l'instant être tenues pour exceptionnels. Comme toutes nos classes d'inférences, pour être ordonnées, doivent contenir des inférences qui ne sont pas purement logiques, mais simplement normales, ces inférences admettent des exceptions qui, tant qu'elles restent des exceptions, ne les mettent pas en cause.

2) DS est une classe d'inférences qui explique en quoi certaines inférences EGA sont discutables. Mais il faut par ailleurs expliquer les motivations des officiels dans leur déclaration. Or cela ne peut se faire par des statistiques, pense Douglas. Les seules données qui nous sont disponibles pour les suicides non reconnus par les officiels, ce sont les patterns socialement significatifs fournis par des déclarations individuelles (journaux, lettres, etc. laissés par les morts), ce sont donc des PSI. Pour rendre compte des exceptions aux EGA introduites par les DS, nous devons donc introduire les PSI. Nous avons donc: $\{$ EGA, IS, RS, DS, PSI \} TM.

3) Une fois introduits ces patterns socialement significatifs fournis par les individus, nous voyons qu'ils nous fournissent aussi des données pour les IS et RS (d'après notre redéfinition des IS et RS). Les classes des IS et des RS comprennent donc aussi des PSI, à côté des PSC (des patterns sociaux d'origine proprement collective). Mais ces PSI ne sont nullement des exceptions dans les formes d'intégration sociale des individus, les IS, même si l'on peut encore les considérer comme exceptionnels dans les RS (les régulations normatives). Si nous ne pouvons pas les considérer comme des exceptions dans une classe qui est au second rang, nous ne pouvons plus leur donner le dernier rang. C'est là une première méta-inférence portant sur les exceptions et normalités. Ils passent même avant les DS, puisqu'ils expliquent aussi la part que l'on peut expliquer des déclarations des DS. Nous avons donc l'ordre: \{EGA, IS, RS et PSI, DS \}TM.

4) Mais à présent, nous pouvons faire une deuxième méta-inférence. Puisque les PSI ne sont pas exceptionnels, les biais introduits par les DS qui sont euxmêmes expliqués principalement par les PSI ne sont pas non plus réductibles à des exceptions. Or les EGA étaient présentées par Durkheim comme donnant une accessibilité immédiate aux faits collectifs, à la réalité du social. Mais si les biais des statistiques ne sont plus exceptionnels, les EGA ne nous donnent pas une telle accessibilité directe. Nous devons contrôler ce qu'ils proposent par d'autres sources. Le rang des EGA doit donc être dégradé. Ils ne sont pas notre classe d'explication optimale. En revanche nous pouvons rehausser le rang des IS, puisque les explications par l'intégration restent compatibles avec les PSI et DS, donc avec ce qui diminue l'importance des EGA, alors même qu'elles sont aussi compatibles avec les EGA. Nous avons donc l'ordre \{IS, EGA, RS et PSI, DS $\}$ TM.

5) Troisième méta-inférence: si les EGA ne sont plus dominantes, alors ce sont bien des explications abstraites, mais elles ne sont plus générales. C'est donc seulement dans certains domaines que les EGA resteront des EGA. Cela leur donne un rang encore inférieur, puisque ces domaines où leur généralité restera inentamée sont normalement des exceptions. Nous avons donc l'ordre: $\{$ IS, RS et PSI, EGA et DS $\}$ TM. 
6) Est-ce que RS mérite ce second rang? Nous nous apercevons que non, parce que les PSI (les motivations individuelles évoquées par les individus tentés par le suicide, y compris ceux qui n'ont pas été déclarés suicidés) sont très souvent en conflit avec les normes sociales qui justement récusent le suicide. Cela nous amène à intervertir le rang des PSI et des RS. Nous avons donc l'ordre:\{IS, PSI, RS, EGA et DS \}TM.

7) Nous pouvons alors tenir compte de la critique que fait Douglas des explications par les intégrations sociales. Elles ne tiennent pas assez compte des motivations contextuelles, dont rendent compte en revanche les PSI. Nous intervertissons alors encore les rangs, et nous avons l'ordre: \{PSI, IS, RS, EGA et DS \} TM.

8) Mais si nos explications inférentielles prioritaires sont des patterns sociaux tels qu'évoqués par les individus, alors il n'y a plus d'espoir de voir des domaines sociaux où les EGA conservent leur généralité. Or si elles ne la conservent pas, elles sont auto-contradictoires. Il faut donc les exclure. Nous avons donc: $\{$ PSI, IS, RS, DS $\}$ EGA, TM.

9) Si nous voulons arriver à l'ordre tel que nous l'avons extrait de nos analyses, il faut tenir compte du fait que c'est essentiellement par des comportements «officiels» que la pression normative des RS se manifeste. On va donc y retrouver des biais signalés par les DS. Nous ne pourrons donc pas mettre les RS plus haut que les RS. Nous aurons l'ordre: \{PSI, IS, RS et DS \}EGA, TM. Mais l'étape 8 nous suffit pour raisonner sur nos conditions de cohérence.

\section{LES AUTRES CONDITIONS DE COHÉRENCE ET LA NOTION DE DISTANCE ENTRE THÉORIES}

Revenons donc aux conditions de cohérence. La transition entre Durkheim et Douglas violait la condition de préférentialité, parce que la sélection des inférences explicatives de Douglas, sur l'ensemble d'explications possibles envisagées par Durkheim, retenait les PSI, alors que Durkheim les rejetait. Nous voyons que dès la deuxième étape de notre chemin de transition entre Durkheim et Douglas, nous avons pu introduire les PSI. Nous avons donc très rapidement pu justifier cette violation de la condition de préférentialité.

En principe, plus une condition est forte, plus sa violation devrait demander un grand nombre d'étapes pour arriver à la justifier. En effet, si la condition est faible, il est certes plus difficile de la violer, mais il plus facile de trouver une justification qui permette de réparer cette violation. Et si elle est forte, il est plus facile de la violer, mais plus difficile de justifier cette violation, puisque les exigences de la condition pour une justification sont plus élevées.

Considérons d'autres conditions de cohérence, plus exigeantes. La plus forte est la condition de Monotonie Rationnelle, ou encore, en théorie du choix social, la condition $\beta$, qui est voisine, ou encore, selon Aleskerov, l'axiome de choix d'Arrow. La propriété $\beta$ dit que si $S \subseteq S$ ', et si $f\left(S^{\prime}\right) \cap S$ est différent de 0 , alors $f(S)$ $\subseteq \mathrm{f}\left(\mathrm{S}^{\prime}\right) \cap \mathrm{S}$. La Monotonie rationnelle et l'axiome de choix d'Arrow exigent que $\mathrm{f}(\mathrm{S})=\mathrm{f}\left(\mathrm{S}^{\prime}\right) \cap \mathrm{S}$. 
Ces conditions sont donc violées si la théorie de Durkheim retient dans sa sélection une classe d'explications que la théorie de Douglas, quand on applique sa sélection aux classes d'explications offertes au choix de Durkheim, ne retient pas. Or nous avons vu que Douglas élimine les EGA, alors que Durkheim les retient. La transition de Durkheim à Douglas viole donc la condition de Monotonie rationnelle.

Nous pouvons vérifier que notre chemin de transition justifiée, étapes par étapes, ne parvient à exclure les EGA qu'à l'étape 8 . Notre chemin de transition justifiée respect donc bien pour l'instant la gradation d'exigence entre les conditions de cohérence.

Terminons en envisageant une troisième condition, celle de Cumulativité, qui correspond en théorie du choix social à la condition nommée en théorie du choix social, axiome d'Aizerman, ou encore par Askerlov condition «Outcast» (de proscription). Elle nous dit que si $\mathrm{f}\left(\mathrm{S}^{\prime}\right) \subseteq \mathrm{S} \subseteq \mathrm{S}^{\prime}$, alors $\mathrm{f}(\mathrm{S}) \subseteq \mathrm{f}\left(\mathrm{S}^{\prime}\right)$ (Aizerman) ou que $\mathrm{f}(\mathrm{S})=\mathrm{f}\left(\mathrm{S}^{\prime}\right)$ (Cumulativité et Outcast).

La relation entre Durkheim et Douglas ne permet pas de satisfaire l'antécédent de cette condition, puisque la sélection de Douglas contient DS, qui n'était pas présent dans l'ensemble des explications entre lesquelles Durkheim a fait sa sélection (il aurait pu en tenir compte, mais il n'a même pas envisagé cette hypothèse). Cependant, nous avons vu qu'il nous suffit d'une seule étape de transition pour compenser ce décalage. Supposons ce décalage compensé. Evidemment la condition de Cumulativité reste violée, puisque EGA fait partie de la sélection de Durkheim, et pas de la sélection de Douglas.

Admettons alors de raisonner ainsi: une fois l'antécédent satisfait (par introduction de DS), combien d'étapes faut il parcourir pour justifier la violation de la Cumulativité? 8 étapes moins une, donc 7 étapes. Ainsi le nombre d'étapes nécessaires pour rationaliser la violation de la Cumulativité est inférieur à celui des étapes nécessaires pour rationaliser la violation de la Monotonie rationnelle. Or la condition de Cumulativité, jointe à la condition de Préférentialité, exige une structure d'ordre (un pré-ordre) qui est moins forte que la condition de Monotonie rationnelle jointe à celle de Préférentialité (une structure par rangs étanche, l'incomparabilité entre deux éléments à l'intérieur d'un rang ne permettant jamais, par une suite transitive d'incomparabilités, de nous relier à un élément d'un autre rang). Nous vérifions donc que le nombre d'étapes de notre chemin de transitions justifiées est plus élevé chaque fois que la condition de cohérence violée est plus forte, et qu'il est inférieur chaque fois qu'elle est plus faible.

Plaçons nous, à titre de contre-épreuve, dans un cas hypothétique où EGA pourrait être très vite exclu, parce qu'il aurait été le dernier élément de la sélection $\mathrm{f}(\mathrm{S})$, pour un Durkheim évidemment fictif. C'est une contre-épreuve parce qu'ainsi, nous limitons au maximum le nombre d'étapes nécessaire pour justifier la violation de la condition $\beta$. Supposons que DS suffise, sans introduire d'autre élément, et ce par une alliance inférentielle immédiate avec IS et un raisonnement de méta-inférence: «puisque DS met en jeu des motivations individuelles et contextuelles, nous ne disposons plus de sources pour nos explications générales abstraites, elles n'ont même pas de domaine de validité réduit, donc ce ne sont plus des explications générales, il faut donc les exclure comme ayant un statut incohérent». Il ne faudrait donc que deux étapes (introduire DS et faire la métainférence, pour réduire la violation de la condition $\beta$. Mais la condition $\alpha$ serait 
aussi déjà satisfaite par là même (en deux étapes aussi). Et l'antécédent de CUM serait aussi satisfait: la sélection de la fonction $f\left(S^{\prime}\right)$ - en enlevant DS- sera incluse dans $S$, qui est inclus dans $S^{\prime}$. Le conséquent serait satisfait par inférence, donc dans une deuxième étape, puisque $f\left(S^{\prime}\right)$ - en enlevant DS- ne permet plus d'enlever EGA et que l'on revient ainsi à $\mathrm{f}\left(\mathrm{S}^{\prime}\right)$. Ainsi les violations de toutes nos conditions de cohérence seraient compensées en deux étapes de trans-cohérence. Nous aurions donc un cas limite où toutes les conditions seraient au même rang, dans notre distance de trans-cohérence. Mais dès que nous sortons de ce cas limite, qui tient à ce que la seule introduction d'une nouvelle donnée se lie immédiatement avec l'élément de rang le plus important pour dégrader le rang du dernier élément et exclure de la sélection ce dernier élément, les différences vont réapparaître.

Mais notre processus de transitions justifiées ne permet il pas d'admettre n'importe quelle violation de cohérence, si échevelée soit-elle? Par exemple, est ce que Douglas ne pourrait pas éliminer RS de sa sélection, ou encore mettre DS avant IS, juste après PSI? Eliminer RS n'est pas justifié, puisque les explications de DS doivent recourir aussi à la référence des officiels aux normes affichées par exemple par leur religion. Et comme les DS dépendent en partie des RS, que les $\mathrm{RS}$ ont un rang inférieur à celui des IS, nous ne pouvons pas faire passer les DS à un rang supérieur à celui des IS. Ainsi, notre processus ne peut pas justifier n'importe quoi, et sa procédure par étapes et par méta-inférence sur les changements de normalité à exceptionnalité est assez contraignante.

Notre condition générale de trans-cohérence est qu'à chaque étape, nous faisons au maximum un seul changement sur l'ordre Le premier changement consiste à introduire la nouvelle donnée. Ensuite chaque changement d'ordre est justifié parce que 1) il permet de supprimer des contradictions entre les inférences permises par l'ensemble des éléments sélectionnés dans l'étape précédente, 2) les éléments qui ne sont pas touchés par ces contradictions conservent entre eux leur ordre 3) ces inférences tiennent compte des rangs des prémisses - une conclusion partant de prémisses qui sont toutes de rang inférieur est une exception par rapport à celle partant de prémisses toutes de rang supérieur, mais des rangs croisés de prémisses peuvent conduire à dire qu'une conclusion n'est pas plus normale que l'autre; 4) Nous pouvons développer des méta-inférences qui portent sur la capacité d'une classe d'inférences à tirer des conclusions normales, exceptionnelles, ou ni normales ni exceptionnelles. Quand une classe d'inférence de rang n se révèle, par suite des conflits avec d'autres inférences admises, n'aboutir qu'à des conclusions de portée inférieure à son rang, (exceptionnelles ou ni normale ni exceptionnelles, quand ses conclusions devraient être normales), nous lui donnons le rang n-1, et inversement dans le cas contraire.

Un processus qui progresse étape par étapes selon ces règles justifie des violations de cohérence au bout d'un certain nombre d'étapes. Un nombre d'étapes supérieur indique une distance supérieure entre théories, et donc une moindre cumulativité (au sens généralisé, capable d'accepter non seulement des révisions, mais des révisions des ordres de priorité qui guident les révisions). 


\section{CONCLUSION}

Nous pouvons donc utiliser notre processus de trans-cohérence par étapes comme une forme de distance entre les théories. Si nous comparons trois théories, nous pourrons dire que la théorie T2 est plus éloignée de T1 que la théorie T3, et donc qu'il y a plus de cumulativité entre T1 et T3 qu'entre T1 et T2. Mais introduisons une théorie T4, qui n'a aucun rapport direct avec T1, mais seulement avec, mettons, T2. C'est seulement via T2 que nous pourrons dire que T4 est éloignée de $\mathrm{T} 1$. Nous ne pourrons pas estimer directement la distance entre T4 et T1, puisque cette distance est seulement relative à un chemin de transition justifiée par étapes. Nos distances ne vont pas s'ordonner sur une seule échelle, fut-elle par degrés, et encore moins nous donner une mesure (de nous permettre de comparer la différence entre les distances d1-d2 à la différence d3-d4). Davantage, il est toujours possible de trouver deux chemins différents pour passer de $\mathrm{T} 1$ à $\mathrm{T} 2$, et donc de changer la distance. Le moment où nous arrivons à compenser la violation des conditions de cohérence est un repère important qui peut permettre une mise en parallèle de ces chemins. Il est aussi possible de privilégier le chemin minimal.

Cependant, il est souhaitable pour une discipline que la distance entre deux théories successives soit réduite, et donc que le degré de cumulativité soit fort. Mais en revanche, il n'est pas souhaitable que l'on satisfasse toujours les conditions de cohérence. Cela reviendrait à conserver toujours la théorie initiale et à introduire des explications par exceptions successives. Mais les épicycles ont été dans l'histoire des sciences le premier exemple d'une telle stratégie, qui n'est pas apparue souhaitable. Nous sommes donc conduits à préférer un degré de cumulativité qui soit non pas le plus élevé possible (aucune violation des conditions de cohérence, ou même se limiter aux violations justifiables en une seule étape de trans-cohérence) mais qui soit un degré de cumulativité modéré. Il semble que parmi les sciences sociales, l'économie ait choisi de privilégier un degré fort de cumulativité, au détriment peut-être de sa capacité à rendre compte de champs expérimentaux non encore explorés. Il est possible que selon les disciplines, on ne puisse pas espérer parvenir à un degré de cumulativité qui dépasse une certaine limite. Le degré de cumulativité de la psychologie expérimentale semble toujours moins élevé que celui de la biologie ou de la physique. Mais ces limitations devraient elles-mêmes être solidement justifiées.

Inversement, la sociologie semble avoir le degré de cumulativité le plus faible. Il est plus faible que celui de l'histoire, car les historiens se soucient de faire une différence entre les documents attestés selon un processus qui présente quelque cumulativité en notre sens, et les théories interprétatives, qui ne sont pas censées présenter un degré de cumulativité aussi fort Cela d'ailleurs nous montre qu'il est tout à fait possible, dans une discipline, que le degré de cumulativité diffère entre le recueil des données, les modèles théoriques utilisés, et les interprétations ou explications proposées. Les auteurs successifs en sociologie semblent avoir eu tendance à majorer la distance entre leur théorie et celles de leurs prédécesseurs. Nous en avons vu un exemple avec une lecture de Douglas qui accentuerait la distance entre lui et Durkheim ${ }^{6}$. Une telle tendance ne devrait pas être encouragée.

- Quand nous avons donné un exemple montrant que notre méthode de justification des transitions ne permet pas de justifier n'importe quoi. 
Une relecture des théories sociologiques qui réduirait leurs distances apparentes parce qu'elle se plierait aux contraintes de notre processus de trans-cohérence par étapes serait donc la bienvenue.

Université de Provence 